\title{
THE RELATIONSHIP BETWEEN LIGHT INTENSITY AND DRY FORAGE YIELD OF COWPEA AND MAIZE UNDER INTERCROPPING
}

(Received: 29. 9. 2011)

\author{
By \\ K.E. Abd-El-Gawad, A.A. Abd-El-Hafeez, W. M. Sharawy* and W. M. El-Nabawy* \\ Department of Agronomy, Faculty of Agriculture, Cairo University \\ * Forage Crops Research Section, FCRI, Agriculture Research Center, Giza, Egypt.
}

\begin{abstract}
Two field experiments were conducted at the Agricultural Experiments and Research Station, Faculty of Agriculture, Cairo University, Giza, Egypt, during 2008 and 2009 summer seasons, to study the intercropping of forage cowpea (Vigna unguiculata L., var. Buff) with maize (Zea mays L.), stay green and single cross hybrid 122 . The experiment was planted at the $1^{s t}$ of July after wheat in both seasons. The experimental design was a split-split plot design arranged in randomized complete blocks with three replicates. The main plots were devoted to three intercropping patterns: 1) Maize on one side of the ridge and forage cowpea on the other side, 2) Solid maize, 3) Solid forage cowpea. Subplots were arranged with plant density of forage cowpea, one and two plants hill ${ }^{-1}$ at $20 \mathrm{~cm}^{2}$ between hills. Sub-sub plots were four nitrogen levels viz., zero, 60,90 and $120 \mathrm{~kg} \mathrm{~N}$ fed. ${ }^{-1} \mathrm{~N}$ was added in two equal doses after 21 and 60 days from planting (after the $1^{\text {st }}$ cut of forage cowpea).

The results indicated significant differences between intercropping patterns, plant density and nitrogen levels for dry yield. Light intensity at top, middle and bottom of cowpea and maize were obtained at each cut in both seasons. Light intensity under solid cowpea was greater for the three levels of light intensity reading than intercropped cowpea plants. At the top of intercropped cowpea plants, light intensity was greater as compared with readings at the middle and bottom of plants. At the middle, light intensity was greater for solid cowpea compared to intercropped cowpea. One plant hill ${ }^{-1}$ was superior to two plants hill ${ }^{-1}$ in light intensity at top, middle and bottom of cowpea plants intercropped with maize at each cut in both seasons. The percentage of increases in light intensity gave lower values between the two plant intensities, it could be recommended with planting two plants hill ${ }^{-1}$ when intercropped cowpea with maize for obtaining high dry forage yield of cowpea. Nitrogen application was significantly decreased light intensity at top, middle and bottom plant of forage cowpea at each cut in both seasons. Nitrogen from zero to $120 \mathrm{~kg} \mathrm{~N}^{-1} \mathrm{~d}^{-1}$ caused reduction in light intensity, While total dry forage yield of cowpea was increased. The highest value of light intensity was obtained by solid cowpea, one plant hill ${ }^{-1}$ and zero nitrogen $\mathrm{fed}^{-1}$, while the lowest value of light intensity was at intercropped cowpea with maize, two plants hill ${ }^{-1}$ and $120 \mathrm{~kg} \mathrm{~N}$ fed. ${ }^{-1}$ For maize plants there were significant differences in light intensity as affected by intercropping patterns at top, middle bottom of maize plants in both seasons except between intercropping two plants hill ${ }^{-1}$ of cowpea with maize and solid maize at top plant before the $1^{s t}$ cut of cowpea in the first season. Nitrogen levels was significantly decreased light intensity at top, middle and bottom plant of maize, with not significant differences between 90 and $120 \mathrm{~kg} \mathrm{~N}$ fed. ${ }^{-1}$ While, grain yield and dry stover yield were increased. Zero $\mathrm{N}$ level had the highest value of light intensity at top, middle and bottom of maize plants compared with the other $\mathrm{N}$ levels. The highest value was obtained by solid maize and zero nitrogen $\mathrm{fed}^{-1}{ }^{-1}$ while the lowest value was at intercropping two plants hill ${ }^{-1}$ of cowpea with maize and $120 \mathrm{~kg} \mathrm{~N}$ fed. $^{-1}$
\end{abstract}

Key words: forage cowpea, intercropping patterns, light intensity, maize, nitrogen levels.

\section{INTRODUCTION}

Growing mixed stands of two or more crop species, defined as intercropping, has many advantages over sole cropping. It provides for efficient utilization of environmental resources, reduces the cost of production, provides greater financial stability for farmers, decreases pest damages, suppresses weed growth, improves soil fertility when legumes are included and improves forage yield and quality (Ofori and Stern, 1987).

Li et al., 2003 indicated that intercropping maximizes the use of the above ground and 
environment resources, including space, light and nutrients, and improve crop yield and quality.

The advantages from intercropping was maximized when the intercropped species complement each other and use the environmental resources more efficiently. Also, they improve the efficiency of using both aboveground and below-ground resources compared to growing the crops as pure stands. The total productivity of an intercropping system is often more than sole cropping of the component crops (Marsalis and Angadi, 2009).

The present investigation aimed at studying the effect of intercropping forage cowpea with maize as a main crop, on light intensity under intercropping, plant density and nitrogen levels to improve forage quality and quantity of intercropped crops for late summer season.

\section{MATERIALS AND METHODS}

Two field trials were carried out during 2008 and 2009 summer seasons at the Agricultural Experiments and Research Station, Faculty of Agriculture, Cairo University, Giza, Egypt. Forage cowpea (Vigna unguiculata L., var. Buff) was grown, using the maize hybrid S.C. 122, which has short stature plant that stay green after grains maturity. The experiments were planted in the $1^{\text {st }}$ of July, following wheat in both seasons, and were designed as split-split plots arranged in randomized complete blocks with three replicates. The main plots were devoted to three intercropping patterns, viz., one side of the ridge for maize and the other side for forage cowpea, solid maize and solid cowpea. Sub-plots were devoted to two plant densities of cowpea i.e., one and two plants hill ${ }^{-1}(30000,60000$ plants/fed) with hills space $20 \mathrm{~cm}$ apart, maize was planted as recommended density (24000 plants/fed) solid or intercropping with forage cowpea with $25 \mathrm{~cm}$ between hills and thinning at one plant hill. ${ }^{-1}$ Sub-sub plots were allotted to four nitrogen levels (Urea 46\%) viz., zero, 60, 90 and $120 \mathrm{~kg}$ $\mathrm{N}$ fed. ${ }^{-1} \mathrm{~N}$ was added in two equal doses after 21 and 60 days from planting (after the $1^{\text {st }}$ cut of cowpea). The experimental unit (sub-sub plot) consisted of 5 ridges, $4 \mathrm{~m}$ long and $70 \mathrm{~cm}$ apart, with area of $14.0 \mathrm{~m}^{2}$ (1/300 fed). Representing soil samples at a depth of $(0-30 \mathrm{~cm})$ were taken before sowing for mechanical and chemical soil analyses in both seasons (Table 1). Forage cowpea was cut twice in both seasons, the $1^{\text {st }}$ cut after 60 days of planting for estimating fresh forage yield of cowpea, while the $2^{\text {nd }}$ one included maize stover mixed with fresh forage from cowpea after 60 days from the $1^{\text {st }}$ cut. Light intensity (Lux) was measured before cutting at the top, middle and bottom $(20 \mathrm{~cm}$ from the soil surface) of forage cowpea and maize plants using a Lux-meter at 12 p.m. Dry yield $\left(\mathrm{t} \mathrm{fed}^{-1}\right)$ of cowpea forage, grain and stover yields of maize $\left(\mathrm{t} \mathrm{fed}^{-1}\right)$ were recorded. Data were analyzed by MSTAT-C Computer program 1986-V4 (Freed, 2005).

Table (1): Mechanical and chemical analyses of soil of the experimental sites before planting in 2008 and 2009.

\begin{tabular}{|l|c|c|}
\hline Mechanical analysis (\%) & $\mathbf{2 0 0 8}$ & $\mathbf{2 0 0 9}$ \\
\hline Coarse sand & 2.1 & 2.4 \\
\hline Fine sand & 24.14 & 23.34 \\
\hline Silt & 18.44 & 20.30 \\
\hline Clay & 47.82 & 46.28 \\
\hline Soil type classification & Clay loam & Clay loam \\
\hline Chemical analysis & & \\
\hline Available N (ppm) & 54 & 51 \\
\hline Available P (ppm) & 26 & 28 \\
\hline \% CaCO & 3.83 & 3.51 \\
\hline \% Organic matter & 1.76 & 1.85 \\
\hline Soil pH & 7.24 & 7.32 \\
\hline
\end{tabular}

\section{RESULTS AND DISCUSSION}

\subsection{Forage cowpea}

\subsubsection{Effect of treatments on light intensity}

Data of light intensity (lux) (Tables 2 to 7 ) show the effect of intercropping patterns, plant density and nitrogen levels at top, middle and bottom of cowpea plants at the $1^{s t}$ and $2^{\text {nd }}$ cut in both seasons.

\subsubsection{Intercropping patterns}

Significant differences were observed in light intensity as affected by intercropping patterns at top, middle and bottom of cowpea plants during the two cuts in both seasons. These results are in agreement with Badr (1998) who demonstrated that light interception by soybean plants was significantly affected by intercropping systems.

Light intensity was greater for solid cowpea at all three levels of plant canopy reading than intercropped cowpea.

Light intensity was the greatest at plant tops as compared with the intensity at the middle and bottom levels. At plant tops light intensity for solid cowpea was $3.4 \%$ and $2.3 \%$ greater than intercropped cowpea at the $1^{s t}$ and $2^{n d}$ cuts in the first season. Light intensity was 3.2\% and 3.3\% greater for the same treatments in respective order, in the second season (Tables 2 and 3).

Light intensity at the middle of the plant was 
greater for solid compared to intercropped cowpea by $8.3 \%$ and $6.8 \%$ at the $1^{s t}$ and $2^{n d}$ cut in the first season, whereas it was greater by $12.1 \%$ and $11.2 \%$ during the same cuts in the second season (Tables 4 and 5).

Light intensity readings at the bottom of the canopy was increased by $14.6 \%$ and $15.1 \%$ for solid cowpea compared to intercropped cowpea at the $1^{\text {st }}$ and $2^{\text {nd }}$ cut in the first season, and was greater by $21.2 \%$ and $19.9 \%$ for the same treatments in the second season (Tables 6 and 7).

These data indicate that the increase in light intensity for solid cowpea culture compared to the intercropped cowpea was due to the competition between forage cowpea and maize for light during vegetative growth. Shading increases in intercropped cowpea than solid cowpea culture. These results are in harmony with those obtained by Abdel-Wahab, (2010) who found that light intensity within soybean canopies is significantly affected by the cropping system. Light intensity was higher for solid soybean as compared with intercropped soybean. Light intensity at the middle and the bottom of soybean canopies decreased under intercropping as compared with recommended solid planting, due to shading effects of adjacent maize plants.

\subsubsection{Plant density}

Light intensity was higher for one plant hill ${ }^{-1}$ than two plants hill ${ }^{-1}$ at the top, the middle and the bottom of cowpea canopy intercropped with maize at each cut in both growing seasons.

Light intensity in the first season, increased under one plant hill ${ }^{-1}$ than two plants hill ${ }^{-1}$ at the top level of the canopy by $2.3 \%$ at the $1^{\text {st }}$ cut and $1.3 \%$ at the $2^{\text {nd }}$ cut, whereas in the second season, the percentages of increases were $2.3 \%$ and $2.0 \%$ at the $1^{\text {st }}$ and $2^{\text {nd }}$ cuts, respectively (Tables 2 and $3)$.

At the middle level of plant canopy the increases in light intensity under one plant hill $^{-1}$ compared to two plants hill ${ }^{-1}$ were $4.4 \%$ and $4.8 \%$ at the $1^{\text {st }}$ and $2^{\text {nd }}$ cuts in the first season, and $4.9 \%$ and $3.8 \%$, respectively at each cut in the second season (Tables 4 and 5).

At the bottom level of the canopy, light intensity was higher under one plant hill ${ }^{-1}$ than two plants hill ${ }^{-1}$ by $8.1 \%$ and $6.3 \%$ at the $1^{\text {st }}$ and $2^{\text {nd }}$ cuts, respectively in the first season and by $8.9 \%$ and $8.8 \%$, respectively at each cut in the second season (Tables 6 and 7).

It was clear that the percentages of increases in light intensity were lower between the two plant intensities. Thus, it could be recommended to plant two cowpea plants hill ${ }^{-1}$ when cowpea is intercropped with maize in order to obtain high dry forage yield of cowpea.

\subsubsection{Nitrogen levels}

Nitrogen application significantly decreased light intensity at top, middle and bottom levels of cowpea canopy at each cut in both seasons.

Under zero $\mathrm{N}$ fertilization light intensity was higher at plant tops compared with $\mathrm{N}$ treatments $\left(60,90\right.$ and $\left.120 \mathrm{~kg} \mathrm{~N}^{-1} \mathrm{fed}^{-1}\right)$, being 1.6, 2.6 and $3.7 \%$ higher at the $1^{\text {st }}$ cut, 2.6, 3.5 and $5.8 \%$ higher at the $2^{\text {nd }}$ cut in the first season. Corresponding increases were 2.2, 3.4 and $4.3 \%$ at the $1^{\text {st }}$ cut, and $1.4,3.1$ and $5.5 \%$ at the $2^{\text {nd }}$ cut in the second season (Tables 2 and 3 ).

At the middle level of the canopy, zero fertilizer treatment showed the highest value of light intensity compared with 60,90 and $120 \mathrm{~kg} \mathrm{~N}$ fed. $^{-1}$ these increases as percentage were 8.2, 12.4 and $16.4 \%$ at the $1^{\text {st }}$ cut, 4.6, 9.9 and $21.9 \%$ at the second one in the first season. In the second season, these increases were 9.0, 16.4 and $24.9 \%$, by $9.5,17.0$ and $24.9 \%$ at the $1^{\text {st }}$ and $2^{\text {nd }}$ cuts, respectively (Tables 4 and 5).

The check treatment $\left(0 \mathrm{~kg} \mathrm{~N} \mathrm{fed}^{-1}\right)$ showed the highest level of light intensity at the bottom level of the canopy reaching 10.5, 19.4 and $24.0 \%$ at the $1^{\text {st }}$ cut, and $10.0,16.0$ and $21.7 \%$ at the second one in the first season. Also, increases were 6.2, 20.6 and $28.2 \%$ at the $1^{\text {st }}$ cut, 7.6, 19.2 and $29.6 \%$ at the $2^{\text {nd }}$ cut in the second season, respectively (Tables 6 and 7).

Applying nitrogen at $120 \mathrm{~kg} \mathrm{~N}^{-\mathrm{fed}^{-1}}$ reduced light intensity, while increased total dry forage yield of cowpea. This may be due to shading effect of maize on cowpea plants and consequently light interception. These results are in agreement with those obtained by Bowes et al., (1970) who found that plant height of soybean increased in response to reduced light intensity. Moreover, Abou Keriasha et al., (2009) found that cowpea intercropped with maize could be attributed to shading effect by maize plants and hence a low amount of intercepted light by cowpea plants. This shading effect increased stem elongation resulting in the tallest plants were observed when cowpea was intercropped with maize on all ridges.

\subsubsection{Effect of the interaction}

The interaction of intercropping patterns, plant density and nitrogen levels influenced light intensity on cowpea intercropped with maize, with the highest value obtained by solid cowpea, one plant hill ${ }^{-1}$ and zero $\mathrm{N}$ fed., ${ }^{-1}$ while the lowest 
Table (2): Effect of intercropping pattern, plant density and nitrogen application on light intensity (lux) at the top of forage cowpea canopy in 2008.

\begin{tabular}{|c|c|c|c|c|c|c|c|c|c|c|c|}
\hline \multirow{3}{*}{$\begin{array}{c}\text { Intercropping } \\
\text { Pattern }\end{array}$} & \multirow{3}{*}{$\begin{array}{l}\text { Plant density } \\
\left(\text { Plant hill }^{-1}\right)\end{array}$} & \multicolumn{5}{|c|}{$1^{\text {st }}$ cut } & \multicolumn{5}{|c|}{$2^{\text {nd }}$ cut } \\
\hline & & \multicolumn{4}{|c|}{ Nitrogen levels $\left(\mathrm{kg} \mathrm{fed}^{-1}\right)$} & \multirow{2}{*}{ Mean } & \multicolumn{4}{|c|}{ Nitrogen levels $\left(\mathrm{kg} \mathrm{fed}^{-1}\right)$} & \multirow{2}{*}{ Mean } \\
\hline & & Zero & 60 & 90 & 120 & & Zero & 60 & 90 & 120 & \\
\hline \multirow{3}{*}{ Solid Cowpea } & One plant & 1398.0 & 1382.0 & 1355.0 & 1345.0 & 1370.0 & 1354.0 & 1317.0 & 1314.0 & 1295.0 & 1320.0 \\
\hline & Two plants & 1368.0 & 1330.0 & 1315.0 & 1287.0 & 1325.0 & 1341.0 & 1310.0 & 1302.0 & 1283.0 & 1309.0 \\
\hline & Mean & 1383.0 & 1356.0 & 1335.0 & 1316.0 & 1347.5 & 1347.5 & 1313.5 & 1308.0 & 1289.0 & 1314.5 \\
\hline \multirow{3}{*}{ Maize +Cowpea } & One plant & 1325.0 & 1311.0 & 1304.0 & 1300.0 & 1310.0 & 1334.0 & 1298.0 & 1285.0 & 1263.0 & 1295.0 \\
\hline & Two plants & 1314.0 & 1295.0 & 1291.0 & 1272.0 & 1293.0 & 1327.0 & 1291.0 & 1270 & 1204.0 & 1273.0 \\
\hline & Mean & 1319.5 & 1303.0 & 1297.5 & 1286.0 & 1301.5 & 1330.5 & 1294.5 & 1277.5 & 1233.5 & 1284.0 \\
\hline \multirow{2}{*}{ General mean } & One plant & 1361.5 & 1346.5 & 1329.5 & 1322.5 & 1340.0 & 1344.0 & 1307.5 & 1299.5 & 1279.0 & 1307.5 \\
\hline & Two plants & 1341.0 & 1312.5 & 1303.0 & 1279.5 & 1309.0 & 1334.0 & 1300.5 & 1286.0 & 1243.5 & 1291.0 \\
\hline \multicolumn{2}{|l|}{ Overall mean } & 1351.3 & 1329.5 & 1316.3 & 1301.0 & 1324.5 & 1339.0 & 1304.0 & 1292.8 & 1261.3 & 1299.3 \\
\hline \multicolumn{12}{|l|}{ L.S.D. at 0.05 level: } \\
\hline \multicolumn{2}{|l|}{ Intercropping pattern (A) } & \multicolumn{5}{|c|}{6.1} & \multicolumn{5}{|c|}{4.9} \\
\hline \multicolumn{2}{|l|}{ Plant density (B) } & \multicolumn{5}{|c|}{--} & \multicolumn{5}{|c|}{---} \\
\hline \multicolumn{2}{|l|}{ Nitrogen levels (C) } & \multirow{2}{*}{\multicolumn{5}{|c|}{$\begin{array}{l}4.3 \\
4.8\end{array}$}} & \multirow{2}{*}{\multicolumn{5}{|c|}{$\begin{array}{l}3.9 \\
4.7\end{array}$}} \\
\hline \multicolumn{2}{|l|}{$\mathrm{A} \times \mathrm{B}$} & & & & & & & & & & \\
\hline \multicolumn{2}{|l|}{$\mathrm{A} \times \mathrm{C}$} & \multicolumn{5}{|c|}{6.0} & \multicolumn{5}{|c|}{5.5} \\
\hline \multirow{2}{*}{\multicolumn{2}{|c|}{$\mathrm{B} \times \mathrm{C}$}} & \multicolumn{5}{|c|}{6.0} & \multicolumn{5}{|c|}{5.5} \\
\hline & & \multicolumn{5}{|c|}{8.5} & \multicolumn{5}{|c|}{7.8} \\
\hline
\end{tabular}

Table (3): Effect of intercropping pattern, plant density and nitrogen application on light intensity (lux) at the top of forage cowpea canopy in 2009.

\begin{tabular}{|c|c|c|c|c|c|c|c|c|c|c|c|}
\hline \multirow{3}{*}{$\begin{array}{l}\text { Intercropping } \\
\text { Pattern }\end{array}$} & \multirow{3}{*}{$\begin{array}{l}\text { Plant density } \\
\left(\text { Plant hill }{ }^{-1}\right)\end{array}$} & \multicolumn{5}{|c|}{$1^{s t} \mathrm{cut}$} & \multicolumn{5}{|c|}{$2^{\text {nd }}$ cut } \\
\hline & & \multicolumn{4}{|c|}{ Nitrogen levels $\left(\mathrm{kg} \mathrm{fed}^{-1}\right)$} & \multirow[b]{2}{*}{ Mean } & \multicolumn{4}{|c|}{ Nitrogen levels $\left(\mathrm{kg} \mathrm{fed}^{-1}\right)$} & \multirow[b]{2}{*}{ Mean } \\
\hline & & Zero & 60 & 90 & 120 & & Zero & 60 & 90 & 120 & \\
\hline \multirow[b]{2}{*}{ Solid Cowpea } & One plant & 1395.0 & 1370.0 & 1351.0 & 1340.0 & 1364.0 & 1373.0 & 1356.0 & 1337.0 & 1314.0 & 1345.0 \\
\hline & Two plants & 1361.0 & 1337.0 & 1321.0 & 1317.0 & 1334.0 & 1362.0 & 1340.0 & 1319.0 & 1291.0 & 1328.0 \\
\hline \multirow{3}{*}{ Maize + Cowpea } & One plant & 1362.0 & 1320.0 & 1315.0 & 1291.0 & 1322.0 & 1348.0 & 1327.0 & 1302.0 & 1267.0 & 1311.0 \\
\hline & Two plants & 1326.0 & 1299.0 & 1270.0 & 1261.0 & 1289.0 & 1311.0 & 1294.0 & 1269.0 & 1226.0 & 1275.0 \\
\hline & Mean & 1344.0 & 1309.5 & 1292.5 & 1276.0 & 1305.5 & 1329.5 & 1310.5 & 1285.5 & 1246.5 & 1293.0 \\
\hline \multirow{2}{*}{ General mean } & One plant & 1378.5 & 1345.0 & 1333.0 & 1315.5 & 1343.0 & 1360.5 & 1341.5 & 1319.5 & 1290.5 & 1328.0 \\
\hline & Two plants & 1343.5 & 1318.0 & 1295.5 & 1289.0 & 1311.5 & 1336.5 & 1317.0 & 1294.0 & 1258.5 & 1301.5 \\
\hline \multicolumn{2}{|l|}{ Overall mean } & 1361.0 & 1331.5 & 1314.3 & 1302.3 & 1327.3 & 1348.5 & 1329.3 & 1306.8 & 1274.5 & 1314.8 \\
\hline \multicolumn{12}{|l|}{ L.S.D. at 0.05 level: } \\
\hline \multicolumn{2}{|l|}{ Intercropping pattern (A) } & \multicolumn{5}{|c|}{4.7} & \multicolumn{5}{|c|}{7.4} \\
\hline \multicolumn{2}{|l|}{ Plant density (B) } & \multirow{2}{*}{\multicolumn{5}{|c|}{---}} & \multirow{2}{*}{\multicolumn{5}{|c|}{$\begin{array}{l}--- \\
5.9\end{array}$}} \\
\hline \multicolumn{2}{|l|}{ Nitrogen levels (C) } & & & & & & & & & & \\
\hline \multicolumn{2}{|l|}{$\mathrm{A} \times \mathrm{B}$} & \multicolumn{5}{|c|}{$\begin{array}{l}4.5 \\
7.0\end{array}$} & \multicolumn{5}{|c|}{7.5} \\
\hline \multicolumn{2}{|l|}{$\mathrm{A} \times \mathrm{C}$} & \multicolumn{5}{|c|}{$\begin{array}{l}7.0 \\
6.4\end{array}$} & \multicolumn{5}{|c|}{8.4} \\
\hline \multirow{2}{*}{\multicolumn{2}{|c|}{$\begin{array}{l}\mathrm{B} \times \mathrm{C} \\
\mathrm{A} \times \mathrm{B} \times \mathrm{C}\end{array}$}} & \multicolumn{5}{|c|}{6.4} & \multicolumn{5}{|c|}{8.4} \\
\hline & & \multicolumn{5}{|c|}{9.1} & \multicolumn{5}{|c|}{11.9} \\
\hline
\end{tabular}


The relationship between light intensity and dry forage yield of cowpea and maize under intercropping.

Table (4): Effect of intercropping pattern, plant density and nitrogen application on light intensity (lux) at the middle level of forage cowpea canopy in 2008 .

\begin{tabular}{|c|c|c|c|c|c|c|c|c|c|c|c|}
\hline \multirow{3}{*}{$\begin{array}{l}\text { Intercropping } \\
\text { Pattern }\end{array}$} & \multirow{3}{*}{$\begin{array}{l}\text { Plant density } \\
\left(\text { Plant hill }^{-1}\right)\end{array}$} & \multicolumn{5}{|c|}{$1^{s t} \mathrm{cut}$} & \multicolumn{5}{|c|}{$2^{\text {nd }}$ cut } \\
\hline & & \multicolumn{4}{|c|}{ Nitrogen levels $\left(\mathrm{kg} \mathrm{fed}^{-1}\right)$} & \multirow{2}{*}{ Mean } & \multicolumn{4}{|c|}{ Nitrogen levels $\left(\mathrm{kg} \mathrm{fed}^{-1}\right)$} & \multirow{2}{*}{ Mean } \\
\hline & & Zero & 60 & 90 & 120 & & Zero & 60 & 90 & 120 & \\
\hline \multirow{3}{*}{ Solid Cowpea } & One plant & 627.0 & 570.0 & 559.0 & 528.0 & 571.0 & 616.0 & 582.0 & 536.0 & 458.0 & 548.0 \\
\hline & Two plants & 588.0 & 545.0 & 523.0 & 512.0 & 542.0 & 551.0 & 532.0 & 520.0 & 449.0 & 513.0 \\
\hline & Mean & 607.5 & 557.5 & 541.0 & 520.0 & 556.5 & 583.5 & 557.0 & 528.0 & 453.5 & 530.5 \\
\hline \multirow{3}{*}{ Maize + Cowpea } & One plant & 584.0 & 531.0 & 502.0 & 463.0 & 520.0 & 547.0 & 524.0 & 496.0 & 441.0 & 502.0 \\
\hline & Two plants & 552.0 & 513.3 & 476.0 & 462.0 & 500.8 & 541.0 & 513.0 & 480.0 & 414.0 & 487.0 \\
\hline & Mean & 568.0 & 522.2 & 489.0 & 462.5 & 510.4 & 544.0 & 518.5 & 488.0 & 427.5 & 494.5 \\
\hline \multirow{2}{*}{ General mean } & One plant & 605.5 & 550.5 & 530.5 & 495.5 & 545.5 & 581.5 & 553.0 & 516.0 & 449.5 & 525.0 \\
\hline & Two plants & 570.0 & 529.2 & 499.5 & 487.0 & 521.4 & 546.0 & 522.5 & 500.0 & 431.5 & 500.0 \\
\hline \multicolumn{2}{|l|}{ Overall mean } & 587.8 & 539.8 & 515.0 & 491.3 & 533.5 & 563.8 & 537.8 & 508.0 & 440.5 & 512.5 \\
\hline \multicolumn{12}{|l|}{ L.S.D. at 0.05 level: } \\
\hline \multicolumn{2}{|l|}{ Intercropping pattern (A) } & \multicolumn{5}{|c|}{3.8} & \multirow{2}{*}{\multicolumn{5}{|c|}{5.2}} \\
\hline \multicolumn{2}{|l|}{ Plant density (B) } & \multicolumn{5}{|c|}{---} & \multirow{2}{*}{\multicolumn{5}{|c|}{$\frac{---}{3.8}$}} \\
\hline \multicolumn{2}{|l|}{ Nitrogen levels (C) } & \multirow{2}{*}{\multicolumn{5}{|c|}{$\frac{4.5}{5.5}$}} & & & & & \\
\hline \multicolumn{2}{|l|}{$\mathrm{A} \times \mathrm{B}$} & & \multirow{2}{*}{\multicolumn{4}{|c|}{5.5}} & \multicolumn{5}{|c|}{7.6} \\
\hline \multicolumn{2}{|l|}{$\mathrm{A} \times \mathrm{C}$} & \multicolumn{2}{|c|}{6.4} & & & & \multicolumn{5}{|c|}{5.4} \\
\hline \multicolumn{2}{|l|}{$\mathrm{B} \times \mathrm{C}$} & \multicolumn{5}{|c|}{6.4} & \multicolumn{5}{|c|}{5.4} \\
\hline $\mathrm{A} \times \mathrm{B} \times \mathrm{C}$ & & & & 9.0 & & & \multicolumn{5}{|c|}{7.6} \\
\hline
\end{tabular}

Table (5): Effect of intercropping pattern, plant density and nitrogen application on light intensity (lux) at the middle level of forage cowpea canopy in 2009.

\begin{tabular}{|c|c|c|c|c|c|c|c|c|c|c|c|}
\hline \multirow{3}{*}{$\begin{array}{l}\text { Intercropping } \\
\text { Pattern }\end{array}$} & \multirow{3}{*}{$\begin{array}{l}\text { Plant density } \\
\left(\text { Plant hill }^{-1}\right)\end{array}$} & \multicolumn{5}{|c|}{$1^{\text {st }} \mathrm{cut}$} & \multicolumn{5}{|c|}{$2^{\text {nd }}$ cut } \\
\hline & & \multicolumn{4}{|c|}{ Nitrogen levels $\left(\mathrm{kg} \mathrm{fed}^{-1}\right)$} & \multirow[b]{2}{*}{ Mean } & \multicolumn{4}{|c|}{ Nitrogen levels $\left(\mathrm{kg} \mathrm{fed}^{-1}\right)$} & \multirow[b]{2}{*}{ Mean } \\
\hline & & Zero & 60 & 90 & 120 & & Zero & 60 & 90 & 120 & \\
\hline \multirow{3}{*}{ Solid Cowpea } & One plant & 662.0 & 608.0 & 576.0 & 534.0 & 595.0 & 637.0 & 581.0 & 538.0 & 516.0 & 568.0 \\
\hline & Two plants & 640.0 & 591.0 & 536.0 & 473.0 & 560.0 & 633.0 & 577.0 & 534.0 & 468.0 & 553.0 \\
\hline & Mean & 651.0 & 599.5 & 556.0 & 503.0 & 577.5 & 635.0 & 579.0 & 536.0 & 492.0 & 560.5 \\
\hline \multirow{3}{*}{ Maize + Cowpea } & One plant & 594.0 & 541.0 & 487.0 & 446.0 & 517.0 & 582.0 & 540.0 & 483.0 & 439.0 & 511.0 \\
\hline & Two plants & 586.0 & 519.0 & 477.0 & 410.0 & 498.0 & 577.0 & 500.0 & 461.0 & 402.0 & 485.0 \\
\hline & Mean & 590.0 & 530.0 & 482.0 & 428.0 & 507.5 & 579.5 & 520.0 & 472.0 & 420.5 & 498.0 \\
\hline \multirow{2}{*}{ General mean } & One plant & 628.0 & 574.0 & 531.5 & 490.0 & 556.0 & 609.5 & 560.5 & 510.5 & 477.5 & 539.5 \\
\hline & Two plants & 613.0 & 555.0 & 506.5 & 441.5 & 529.0 & 605.0 & 538.5 & 497.5 & 435.0 & 519.0 \\
\hline \multicolumn{2}{|l|}{ Overall mean } & 620.5 & 564.8 & 519.0 & 465.8 & 542.5 & 607.3 & 549.5 & 504.0 & 456.3 & 529.3 \\
\hline \multicolumn{12}{|l|}{ L.S.D. at 0.05 level: } \\
\hline \multicolumn{2}{|l|}{ Intercropping pattern (A) } & \multicolumn{5}{|c|}{4.9} & \multicolumn{4}{|c|}{7.8} & \\
\hline \multirow{2}{*}{\multicolumn{2}{|c|}{$\begin{array}{l}\text { Plant density (B) } \\
\text { Nitrogen levels (C) }\end{array}$}} & \multirow{2}{*}{\multicolumn{5}{|c|}{$\begin{array}{l}--- \\
4.5\end{array}$}} & \multirow{2}{*}{\multicolumn{4}{|c|}{$\begin{array}{l}--- \\
4.9 \\
\end{array}$}} & \\
\hline & & & & & & & & & & & \\
\hline \multicolumn{2}{|l|}{$\mathrm{A} \times \mathrm{B}$} & \multicolumn{5}{|c|}{9.3} & \multirow{2}{*}{\multicolumn{4}{|c|}{2.4}} & \\
\hline \multicolumn{2}{|l|}{$\mathrm{A} \times \mathrm{C}$} & \multirow{2}{*}{\multicolumn{5}{|c|}{6.3}} & & & & & \\
\hline \multirow{2}{*}{\multicolumn{2}{|c|}{$\frac{B \times C}{A \times B \times C}$}} & \multirow{2}{*}{\multicolumn{4}{|c|}{$\begin{array}{l}6.3 \\
8.9\end{array}$}} & & \multicolumn{4}{|c|}{6.9} & \\
\hline & & & & & & & \multicolumn{4}{|c|}{9.9} & \\
\hline
\end{tabular}


Table (6): Effect of intercropping pattern, plant density and nitrogen application on light intensity (lux) at the bottom level of forage cowpea canopy in 2008.

\begin{tabular}{|c|c|c|c|c|c|c|c|c|c|c|c|}
\hline \multirow{3}{*}{$\begin{array}{l}\text { Intercropping } \\
\text { Pattern }\end{array}$} & \multirow{3}{*}{$\begin{array}{l}\text { Plant density } \\
\left(\text { Plant hill }^{-1}\right)\end{array}$} & \multicolumn{5}{|c|}{$1^{s t} \mathrm{cut}$} & \multicolumn{5}{|c|}{$2^{\text {nd }}$ cut } \\
\hline & & \multicolumn{4}{|c|}{ Nitrogen levels $\left(\mathrm{kg} \mathrm{fed}^{-1}\right)$} & \multirow{2}{*}{ Mean } & \multicolumn{4}{|c|}{ Nitrogen levels $\left(\mathrm{kg} \mathrm{fed}^{-1}\right)$} & \multirow{2}{*}{ Mean } \\
\hline & & Zero & 60 & 90 & 120 & & Zero & 60 & 90 & 120 & \\
\hline \multirow{3}{*}{ Solid Cowpea } & One plant & 125.0 & 116.0 & 99.0 & 92.0 & 108.0 & 121.0 & 104.0 & 97.0 & 90.0 & 103.0 \\
\hline & Two plants & 112.0 & 98.0 & 91.0 & 87.0 & 97.0 & 108.0 & 100.0 & 90.0 & 86.0 & 96.0 \\
\hline & Mean & 118.5 & 107.0 & 95.0 & 89.5 & 102.5 & 114.5 & 102.0 & 93.5 & 88.0 & 99.5 \\
\hline \multirow{3}{*}{ Maize + Cowpea } & One plant & 105.0 & 91.0 & 84.0 & 80.0 & 90.0 & 96.0 & 88.0 & 84.0 & 80.0 & 87.0 \\
\hline & Two plants & 97.0 & 88.0 & 80.0 & 75.0 & 85.0 & 93.0 & 84.0 & 80.0 & 71.0 & 82.0 \\
\hline & Mean & 101.0 & 89.5 & 82.0 & 77.5 & 87.5 & 94.5 & 86.0 & 82.0 & 75.5 & 84.5 \\
\hline \multirow{2}{*}{ General mean } & One plant & 115.0 & 103.5 & 91.5 & 86.0 & 99.0 & 108.5 & 96.0 & 90.5 & 85.0 & 95.0 \\
\hline & Two plants & 104.5 & 93.0 & 85.5 & 81.0 & 91.0 & 100.5 & 92.0 & 85.0 & 78.5 & 89.0 \\
\hline \multicolumn{2}{|c|}{ Overall mean } & 109.8 & 98.3 & 88.5 & 83.5 & 95.0 & 104.5 & 94.0 & 87.8 & 81.8 & 92.0 \\
\hline \multicolumn{12}{|c|}{ L.S.D. at 0.05 level:- } \\
\hline \multicolumn{2}{|c|}{ Intercropping pattern (A) } & \multicolumn{5}{|c|}{2.9} & \multicolumn{5}{|c|}{1.9} \\
\hline \multicolumn{2}{|l|}{ Plant density (B) } & \multicolumn{5}{|c|}{---} & \multicolumn{5}{|c|}{---} \\
\hline \multicolumn{2}{|l|}{ Nitrogen levels (C) } & \multicolumn{5}{|c|}{2.9} & \multirow{2}{*}{\multicolumn{5}{|c|}{$\frac{2.5}{4.9}$}} \\
\hline \multicolumn{2}{|l|}{$\mathrm{A} \times \mathrm{B}$} & \multirow{2}{*}{\multicolumn{5}{|c|}{$\frac{5.6}{4.2}$}} & & & & & \\
\hline \multicolumn{2}{|l|}{$\mathrm{A} \times \mathrm{C}$} & & & & & & \multicolumn{5}{|c|}{3.6} \\
\hline \multirow{2}{*}{\multicolumn{2}{|c|}{$\mathrm{B} \times \mathrm{C}$}} & \multicolumn{5}{|c|}{4.2} & \multicolumn{5}{|c|}{3.6} \\
\hline & & \multicolumn{5}{|c|}{5.9} & \multicolumn{5}{|c|}{5.1} \\
\hline
\end{tabular}

Table (7): Effect of intercropping pattern, plant density and nitrogen application on light intensity (lux) at the bottom level of forage cowpea canopy in 2009.

\begin{tabular}{|c|c|c|c|c|c|c|c|c|c|c|c|}
\hline \multirow{3}{*}{$\begin{array}{l}\text { Intercropping } \\
\text { pattern }\end{array}$} & \multirow{3}{*}{$\begin{array}{l}\text { Plant density } \\
\left(\text { Plant hill }{ }^{-1}\right)\end{array}$} & \multicolumn{5}{|c|}{$1^{s t} \mathrm{cut}$} & \multicolumn{5}{|c|}{$2^{\text {nd }}$ cut } \\
\hline & & \multicolumn{4}{|c|}{ Nitrogen levels $\left(\mathrm{kg} \mathrm{fed}^{-1}\right)$} & \multirow[b]{2}{*}{ Mean } & \multicolumn{4}{|c|}{ Nitrogen levels $\left(\mathrm{kg} \mathrm{fed}^{-1}\right)$} & \multirow{2}{*}{ Mean } \\
\hline & & Zero & 60 & 90 & 120 & & Zero & 60 & 90 & 120 & \\
\hline \multirow{3}{*}{ Solid Cowpea } & One plant & 131.0 & 123.0 & 107.0 & 99.0 & 115.0 & 126.0 & 118.0 & 100.0 & 92.0 & 109.0 \\
\hline & Two plants & 122.0 & 115.0 & 91.0 & 80.0 & 102.0 & 117.0 & 106.0 & 95.0 & 70.0 & 97.0 \\
\hline & Mean & 126.5 & 119.0 & 99.0 & 89.5 & 108.5 & 121.5 & 112.0 & 97.5 & 81.0 & 103.0 \\
\hline \multirow{3}{*}{ Maize + Cowpea } & One plant & 101.0 & 95.0 & 82.0 & 74.0 & 88.0 & 97.0 & 90.0 & 81.0 & 72.0 & 85.0 \\
\hline & Two plants & 96.0 & 89.0 & 77.0 & 70.0 & 83.0 & 92.0 & 85.0 & 73.0 & 70.0 & 80.0 \\
\hline & Mean & 98.5 & 92.0 & 79.5 & 72.0 & 85.5 & 94.5 & 87.5 & 77.0 & 71.0 & 82.5 \\
\hline \multirow{2}{*}{ General mean } & One plant & 116.0 & 109.0 & 94.5 & 86.5 & 101.5 & 111.5 & 104.0 & 90.5 & 82.0 & 97.0 \\
\hline & Two plants & 109.0 & 102.0 & 84.0 & 75.0 & 92.5 & 104.5 & 95.5 & 84.0 & 70.0 & 88.5 \\
\hline \multicolumn{2}{|c|}{ Overall mean } & 112.5 & 105.5 & 89.3 & 80.8 & 97.0 & 108.0 & 99.8 & 87.3 & 76.0 & 92.8 \\
\hline \multicolumn{12}{|l|}{ L.S.D. at 0.05 level: } \\
\hline \multicolumn{2}{|c|}{ Intercropping pattern (A) } & \multicolumn{5}{|c|}{6.2} & \multicolumn{5}{|c|}{3.1} \\
\hline \multicolumn{2}{|l|}{ Plant density (B) } & \multirow{2}{*}{\multicolumn{5}{|c|}{$\begin{array}{l}--- \\
2.8\end{array}$}} & \multirow{2}{*}{\multicolumn{5}{|c|}{$\begin{array}{l}--- \\
3.3\end{array}$}} \\
\hline \multicolumn{2}{|l|}{ Nitrogen levels (C) } & & & & & & & & & & \\
\hline \multicolumn{2}{|l|}{$\mathrm{A} \times \mathrm{B}$} & \multicolumn{5}{|c|}{4.2} & \multicolumn{5}{|c|}{1.7} \\
\hline \multicolumn{2}{|l|}{$\mathrm{A} \times \mathrm{C}$} & \multicolumn{5}{|c|}{4.1} & \multicolumn{5}{|c|}{4.7} \\
\hline \multirow{2}{*}{\multicolumn{2}{|c|}{$\begin{array}{l}\mathrm{B} \times \mathrm{C} \\
\mathrm{A} \times \mathrm{B} \times \mathrm{C}\end{array}$}} & \multicolumn{5}{|c|}{4.1} & \multicolumn{5}{|c|}{4.7} \\
\hline & & \multicolumn{5}{|c|}{5.8} & \multicolumn{5}{|c|}{6.7} \\
\hline
\end{tabular}


value of light intensity was noted for cowpea intercropped with maize at two plants hill $^{-1}$ and fertilized by $120 \mathrm{~kg} \mathrm{~N} \mathrm{fed}^{-1}$

\subsubsection{The effect of light intensity on cowpea dry forage yield}

Intercropping patterns significantly affected the dry forage yield of cowpea. The average dry forage yield $\left(\mathrm{t} \mathrm{fed}^{-1}\right)$ of cowpea intercropped with maize and solid cowpea reached 1.0 and $1.1 \mathrm{t} \mathrm{fed}^{-1}$ 1.4 and $1.6 \mathrm{t} \mathrm{fed}^{-1}$ at the $1^{\text {st }}$ and $2^{\text {nd }}$ cuts, in the first season. Corresponding values for the second season were 0.8 and $1.0 \mathrm{t} \mathrm{fed}^{-1} 1.1$ and $1.3 \mathrm{t} \mathrm{fed}^{-1}$ at the $1^{s t}$ and $2^{\text {nd }}$ cut, respectively (Tables 8 and 9). It was clear that solid cowpea gave the highest dry forage yield because light intensity at plant tops of solid cowpea was greater by $3.4 \%$ and $2.3 \%$ compared to intercropped cowpea at the $1^{s t}$ and $2^{\text {nd }}$ cuts in the first season. While, corresponding increases were $3.2 \%$ and $3.3 \%$ in the second season (Tables 2 and 3). Also, light intensity at the middle of the canopy increase for solid cowpea compared to intercropped cowpea by $8.3 \%$ and $6.8 \%$ at the $1^{\text {st }}$ and $2^{\text {nd }}$ cut in the first season, and by $12.1 \%$ and $11.2 \%$ for the same cuts in the second season (Tables 4 and 5). Moreover, bottom reading of light intensity increased by $14.6 \%$ and $15.1 \%$ for solid cowpea compared to intercropped cowpea at the $1^{\text {st }}$ and $2^{\text {nd }}$ cut, in the first season. Also, corresponding increases in the second season were $21.2 \%$ and $19.9 \%$ at the $1^{s t}$ and $2^{n d}$ cut (Tables 6 and 7). Therefore, shading increased in cowpea intercropped with maize than solid cowpea. These results are in agreement with ElZanaty, (2006) who reported that dry forage yield of cowpea in pure stands significantly surpassed its yield under intercropping in both seasons.

Two plants hill ${ }^{-1}$ gave the highest dry forage yield compared with one plant hill ${ }^{-1}$ at each cut in both seasons. However, the light intensity at one plant hill ${ }^{-1}$ was lower than two plants hill ${ }^{-1}$ at each cut for top, middle and bottom in both seasons. So it could be recommended with planting two plants hill $^{-1}$ when intercropped cowpea and maize for obtaining high dry forage yield of cowpea.

Dry forage yield was significantly affected by nitrogen levels below 90 and $120 \mathrm{~kg} \mathrm{~N}^{-1} \mathrm{fed}^{-1}$ applied at the $2^{\text {nd }}$ cut in both seasons. The average dry forage yield $\left(\mathrm{t} \mathrm{fed}^{-1}\right)$ of cowpea supplied with zero to $120 \mathrm{~kg} \mathrm{~N}$ fed $^{-1}$ recorded 0.6 and $1.3 \mathrm{t} \mathrm{fed}^{-1}$ 1.0 and $1.8 \mathrm{t} \mathrm{fed}^{-1}$ at the $1^{\text {st }}$ and $2^{\text {nd }}$ cut in the first season. Also, it was 0.5 and $1.1 \mathrm{t} \mathrm{fed}^{-1} 0.8$ and 1.5 $\mathrm{t} \mathrm{fed}^{-1}$ in the second season for the same treatments. On the other hand, the control treatment (zero $\mathrm{N}$ ) was superior than other nitrogen levels $\left(60,90\right.$ and $120 \mathrm{~kg} \mathrm{~N}$ fed $\left.^{-1}\right)$ in light intensity at the bottom level. These increases were 10.5, 19.4 and $24.0 \%$ at the $1^{\text {st }}$ cut, and 10.0, 16.0 and $21.7 \%$ at the $2^{\text {nd }}$ cut in the first season. Also, corresponding increases in the second season were 6.2, 20.6 and $28.2 \%$ at the $1^{\text {st }}$ cut, and 7.6, 19.2 and $29.6 \%$ at the $2^{\text {nd }}$ cut. The interaction between intercropped cowpea with maize at two plants hill ${ }^{1}$ with the addition of $90 \mathrm{~kg} \mathrm{~N} \mathrm{fed}^{-1}$ gave $3.6 \mathrm{t} \mathrm{fed}^{-1}$ for total dry forage yield, while solid cowpea in the same treatment was $4.1 \mathrm{t} \mathrm{fed.}^{-1}$ in the first season without significant effects between the two levels 90 and $120 \mathrm{~kg} \mathrm{~N}$ fed $^{-1}$ (Tables 8 and 9).

\subsection{Intercropped maize}

\subsubsection{Effect of different treatments on light intensity}

Light intensity (Lux) at the top, middle and bottom of intercropped maize plants before the $1^{\text {st }}$ and $2^{\text {nd }}$ cuts of cowpea was significantly affected by intercropping patterns and applied nitrogen levels in the first and second seasons.

\subsubsection{Intercropping patterns}

There were significant differences in light intensity at the top, middle and the bottom level of maize plants due to intercropping patterns before the $1^{\text {st }}$ and $2^{\text {nd }}$ cuts of cowpea in both seasons, except between intercropping two plants of cowpea hill ${ }^{-1}$ with maize and solid maize at top plant before the $1^{\text {st }}$ cut of cowpea in the first and second seasons and the $2^{\text {nd }}$ cut of cowpea in the first season. These results are in agreement with those obtained by Metwally et al., (2005) who mentioned that maize canopy architecture (spatial distribution of shoot organs) plays an important role in the amount of sunlight radiation intercepted by soybean under intercropping.

On the other hand, light intensity at the top of the plants of intercropped maize before the $2^{\text {nd }}$ cut of cowpea was not significantly affected by intercropping patterns, except for intercropping one plant hill ${ }^{-1}$ of cowpea with maize and solid maize in the first season, while, it was significant in the second season except between intercropping one plant hill $^{-1}$ of cowpea with maize and intercropping two plants hill ${ }^{-1}$ cowpea of with maize in the second season.

In the first season, light intensity at the top of maize plants before the $1^{\text {st }}$ cut of cowpea (one plant hill $^{-1}$ ) increased by $0.3 \%$ compared with solid maize. However, light intensity of solid maize increased by $0.1 \%$ compared to intercropping two plants hill ${ }^{-1}$ of cowpea with maize (Tables 10 and 11).

In the second season at the $1^{\text {st }}$ cut, solid maize 
Table (8): Dry forage yield ( $\left(\right.$ fed $\left.^{-1}\right)$ for $1^{\text {st }}$ and $2^{\text {nd }}$ cuts of forage cowpea as affected by intercropping pattern, plant density and nitrogen application in 2008.

\begin{tabular}{|c|c|c|c|c|c|c|c|c|c|c|c|}
\hline \multirow{3}{*}{$\begin{array}{l}\text { Intercropping } \\
\text { Pattern }\end{array}$} & \multirow{3}{*}{$\begin{array}{c}\text { Plant density } \\
\left(\text { Plant hill }{ }^{-1}\right)\end{array}$} & \multicolumn{5}{|c|}{$1^{s t}$ cut } & \multicolumn{5}{|c|}{$2^{\text {nd }}$ cut } \\
\hline & & \multicolumn{4}{|c|}{ Nitrogen levels $\left(\mathrm{kg} \mathrm{fed}^{-1}\right)$} & \multirow[b]{2}{*}{ Mean } & \multicolumn{4}{|c|}{ Nitrogen levels $\left(\mathrm{kg} \mathrm{fed}^{-1}\right)$} & \multirow[b]{2}{*}{ Mean } \\
\hline & & Zero & 60 & 90 & 120 & & Zero & 60 & 90 & 120 & \\
\hline \multirow{3}{*}{ Solid Cowpea } & One plant & 0.6 & 0.8 & 1.3 & 1.0 & 0.9 & 0.8 & 1.3 & 1.5 & 1.6 & 1.3 \\
\hline & Two plants & 0.8 & 1.1 & 1.8 & 1.8 & 1.4 & 1.3 & 1.7 & 2.3 & 2.2 & 1.9 \\
\hline & Mean & 0.7 & 1.0 & 1.5 & 1.4 & 1.1 & 1.1 & 1.5 & 1.9 & 1.9 & 1.6 \\
\hline \multirow{3}{*}{ Maize + Cowpea } & One plant & 0.5 & 0.7 & 1.0 & 0.9 & 0.8 & 0.7 & 0.9 & 1.4 & 1.5 & 1.1 \\
\hline & Two plants & 0.7 & 1.2 & 1.6 & 1.4 & 1.2 & 1.1 & 1.5 & 2.0 & 1.9 & 1.6 \\
\hline & Mean & 0.6 & 0.9 & 1.3 & 1.2 & 1.0 & 0.9 & 1.2 & 1.7 & 1.7 & 1.4 \\
\hline \multirow{2}{*}{ General mean } & One plant & 0.5 & 0.7 & 1.1 & 1.0 & 0.8 & 0.8 & 1.1 & 1.4 & 1.6 & 1.2 \\
\hline & Two plants & 0.8 & 1.1 & 1.7 & 1.6 & 1.3 & 1.2 & 1.6 & 2.1 & 2.0 & 1.7 \\
\hline \multicolumn{2}{|c|}{ Overall mean } & 0.6 & 0.9 & 1.4 & 1.3 & 1.1 & 1.0 & 1.3 & 1.8 & 1.8 & 1.5 \\
\hline \multicolumn{12}{|l|}{ L.S.D. at 0.05 level: } \\
\hline \multicolumn{2}{|c|}{ Intercropping pattern (A) } & \multicolumn{5}{|c|}{0.01} & \multicolumn{5}{|c|}{0.05} \\
\hline \multirow{2}{*}{\multicolumn{2}{|c|}{$\begin{array}{c}\text { Plant density (B) } \\
\text { Nitrogen levels (C) }\end{array}$}} & \multicolumn{5}{|c|}{---} & \multicolumn{5}{|c|}{---} \\
\hline & & \multirow{2}{*}{\multicolumn{5}{|c|}{$\begin{array}{l}0.05 \\
0.05\end{array}$}} & \multicolumn{5}{|c|}{0.06} \\
\hline \multicolumn{2}{|c|}{$\frac{\text { Nitrogen levels (C) }}{\text { A x B }}$} & & & & & & \multirow{2}{*}{\multicolumn{5}{|c|}{$\begin{array}{l}0.10 \\
0.08\end{array}$}} \\
\hline \multicolumn{2}{|c|}{$\mathrm{AxC}$} & \multicolumn{5}{|c|}{0.06} & & & & & \\
\hline \multicolumn{2}{|c|}{$\mathrm{B} \times \mathrm{C}$} & \multirow{2}{*}{\multicolumn{5}{|c|}{0.06}} & \multicolumn{5}{|c|}{0.08} \\
\hline \multicolumn{2}{|c|}{$\mathrm{A} \times \mathrm{B} \times \mathrm{C}$} & & & & & & \multicolumn{5}{|c|}{0.12} \\
\hline
\end{tabular}

Table (9): Dry forage yield $\left(\mathrm{t} \mathrm{fed}^{-1}\right)$ for $\mathbf{1}^{\text {st }}$ and $2^{\text {nd }}$ cuts of forage cowpea as affected by intercrop pingpattern, plant density and nitrogen application in 2009.

\begin{tabular}{|c|c|c|c|c|c|c|c|c|c|c|c|}
\hline \multirow{3}{*}{$\begin{array}{l}\text { Intercropping } \\
\text { Pattern }\end{array}$} & \multirow{3}{*}{$\begin{array}{l}\text { Plant density } \\
\left(\text { (Plant hill }^{-1}\right)\end{array}$} & \multicolumn{5}{|c|}{$1^{s t}$ cut } & \multicolumn{5}{|c|}{$2^{\text {nd }}$ cut } \\
\hline & & \multicolumn{4}{|c|}{ Nitrogen levels $\left(\mathrm{kg} \mathrm{fed}^{-1}\right)$} & \multirow[t]{2}{*}{ Mean } & \multicolumn{4}{|c|}{ Nitrogen levels $\left(\mathrm{kg} \mathrm{fed}^{-1}\right)$} & \multirow{2}{*}{ Mean } \\
\hline & & Zero & 60 & 90 & 120 & & Zero & 60 & 90 & 120 & \\
\hline \multirow{3}{*}{ Solid Cowpea } & One plant & 0.5 & 0.7 & 1.1 & 0.9 & 0.8 & 0.7 & 1.0 & 1.2 & 1.3 & 1.1 \\
\hline & Two plants & 0.7 & 0.9 & 1.5 & 1.5 & 1.1 & 1.1 & 1.4 & 1.9 & 1.8 & 1.6 \\
\hline & Mean & 0.6 & 0.8 & 1.3 & 1.2 & 1.0 & 0.9 & 1.2 & 1.6 & 1.6 & 1.3 \\
\hline \multirow{3}{*}{ Maize + Cowpea } & One plant & 0.4 & 0.5 & 0.9 & 0.8 & 0.6 & 0.6 & 0.8 & 1.2 & 1.3 & 0.9 \\
\hline & Two plants & 0.6 & 1.0 & 1.3 & 1.2 & 1.0 & 0.9 & 1.2 & 1.7 & 1.6 & 1.3 \\
\hline & Mean & 0.5 & 0.8 & 1.1 & 1.0 & 0.8 & 0.7 & 1.0 & 1.4 & 1.4 & 1.1 \\
\hline \multirow[b]{2}{*}{ General mean } & One plant & 0.4 & 0.6 & 1.0 & 0.8 & 0.7 & 0.6 & 0.9 & 1.2 & 1.3 & 1.0 \\
\hline & Two plants & 0.6 & 0.9 & 1.4 & 1.3 & 1.1 & 1.0 & 1.3 & 1.8 & 1.7 & 1.5 \\
\hline \multicolumn{2}{|c|}{ Overall mean } & 0.5 & 0.8 & 1.2 & 1.1 & 0.9 & 0.8 & 1.1 & 1.5 & 1.5 & 1.2 \\
\hline \multicolumn{12}{|l|}{ L.S.D. at 0.05 level: } \\
\hline \multicolumn{2}{|c|}{ Intercropping pattern (A) } & \multirow{2}{*}{\multicolumn{5}{|c|}{0.01}} & \multicolumn{5}{|c|}{0.04} \\
\hline \multirow{2}{*}{\multicolumn{2}{|c|}{$\begin{array}{c}\text { Plant density (B) } \\
\text { Nitrogen levels (C) }\end{array}$}} & & & & & & \multirow{2}{*}{\multicolumn{5}{|c|}{---}} \\
\hline & & \multicolumn{5}{|c|}{0.04} & & & & & \\
\hline \multicolumn{2}{|c|}{$\mathrm{A} \times \mathrm{B}$} & \multicolumn{5}{|c|}{0.04} & \multicolumn{5}{|c|}{0.09} \\
\hline \multicolumn{2}{|c|}{$\mathrm{A} \times \mathrm{C}$} & \multirow{2}{*}{\multicolumn{5}{|c|}{0.05}} & \multicolumn{5}{|c|}{0.07} \\
\hline \multicolumn{2}{|c|}{$\mathrm{B} \times \mathrm{C}$} & \multirow{2}{*}{\multicolumn{2}{|c|}{0.05}} & & & & \multicolumn{5}{|c|}{0.07} \\
\hline \multicolumn{2}{|c|}{$\mathrm{A} \times \mathrm{B} \times \mathrm{C}$} & \multicolumn{4}{|c|}{0.08} & & \multicolumn{5}{|c|}{0.10} \\
\hline
\end{tabular}


increased by $0.1 \%$ compared with intercropping one plant hill $^{-1}$ of cowpea with maize, and by $0.4 \%$ as compared with intercropping two plants hill $^{-1}$ of cowpea with maize (Tables 10 and 11).

On the other hand, light intensity of intercropped maize before the $2^{\text {nd }}$ cut of cowpea increased for solid maize by $0.4 \%$ as compared with intercropping one plant hill ${ }^{-1}$ of cowpea with maize, by $0.1 \%$ as compared with intercropping two plants hill ${ }^{-1}$ of cowpea with maize in the first season. Also, it was increased by $0.2 \%$ and $0.3 \%$ for the same respective treatments in the second season (Tables 10 and 11).

Light intensity at the middle level of maize, before the $1^{\text {st }}$ cut of cowpea in the first season was greater for solid maize than intercropped maize by $3.3 \%$ and $5.0 \%$ when intercropping one plant hill ${ }^{-1}$ and two plants hill $^{-1}$ of cowpea with maize, respectively. Corresponding of increases were $3.5 \%$ and $5.9 \%$ for the same treatments in respective order, in the second season. Light intensity increased by $2.5 \%$ and $4.5 \%$ for solid maize over maize intercropped at one plant hill ${ }^{-1}$ and two plants hill ${ }^{-1}$ of cowpea before the $2^{\text {nd }}$ cut in the first season, and by $4.1 \%$ and $6.1 \%$ for the same treatments in the same order in the second season (Tables 12 and 13).

On the other hand, bottom reading of light intensity increased by $6.1 \%$ and $12.1 \%$ for solid maize compared to maize intercropped with one and two plants hill ${ }^{-1}$ of cowpea before the $1^{\text {st }}$ cut of cowpea in the first season, and was increased by $8.0 \%$ and $10.7 \%$ for the same treatments in the second season.

Light intensity readings at the bottom of maize canopy before the $2^{\text {nd }}$ cut of cowpea was greater by $6.8 \%$ and $12.3 \%$ for solid maize compared to intercropping one plant and two plants hill ${ }^{-1}$ of cowpea with maize in the first season. Corresponding increases were $7.6 \%$ and $10.0 \%$ in the second season (Tables 14 and 15).

\subsubsection{Nitrogen levels}

The addition of nitrogen significantly decreased light intensity at the top, middle and bottom levels of maize before the $1^{\text {st }}$ and $2^{\text {nd }}$ cuts of cowpea in both seasons, except between the two levels 90 and $120 \mathrm{~kg} \mathrm{~N}^{-1} \mathrm{de}^{-1}$ at top and middle reading before the $1^{\text {st }}$ and $2^{\text {nd }}$ cuts of cowpea in the first season, respectively.

Zero fertilizer treatment had the highest value of light intensity at the top of maize plants compared with the applied $\mathrm{N}$ levels $(60,90$ and $120 \mathrm{~kg} \mathrm{~N} \mathrm{fed}^{-1}$ ), reaching $0.5,0.9$ and $1.1 \%$ before the $1^{\text {st }}$ cut of cowpea, and $0.5,0.8$ and $1.2 \%$ before the $2^{\text {nd }}$ cut of cowpea in the first season. Corresponding values in the second season were $0.3,0.7$ and $1.2 \%$ before the $1^{\text {st }}$ cut of cowpea, and $0.4,0.8$ and $1.6 \%$ before the $2^{\text {nd }}$ cut of cowpea, respectively (Tables 10 and 11).

On the other hand, at the middle level of maize plants, zero fertilizer treatments showed the highest value of light intensity compared with the 60,90 and $120 \mathrm{~kg} \mathrm{~N}^{-1}$ treatments, these increases in light intensity were 1.6, 3.0 and $4.1 \%$ before the $1^{\text {st }}$ cut, and 1.4, 2.9 and $3.3 \%$ before the $2^{\text {nd }}$ cut of cowpea in the first season. Corresponding increases were 1.8, 3.5 and $5.0 \%$ before the $1^{\text {st }}$ cut of cowpea, and 1.7, 3.2 and 4.0\% before the $2^{\text {nd }}$ cut of cowpea, in the second season respectively (Tables 12 and 13).

Zero $\mathrm{N}$ fertilizer treatment was superior in light intensity to nitrogen added levels at the 60,90 and $120 \mathrm{kgNfed}^{-1}$ at the bottom of maize plant, showing increases of 7.0, 11.6 and $16.7 \%$ before the $1^{\text {st }}$ cut of cowpea, and 4.7, 8.0 and $12.7 \%$ before the $2^{\text {nd }}$ cut in the first season. Corresponding increases in the second season were 6.4, 10.2 and $15.5 \%$ before the $1^{\text {st }}$ cut, and 5.2, 9.3 and $13.8 \%$ before the $2^{\text {nd }}$ cut of cowpea in the second season, respectively (Tables 14 and 15).

The application of nitrogen up to $120 \mathrm{~kg} \mathrm{~N} \mathrm{fed}^{-1}$ caused increased reduction in light intensity. As a result, grain yield and dry stover yield were increased.

\subsubsection{Effect of the interaction between intercropping patterns and $\mathrm{N}$ levels}

The interaction effect of intercropping patterns and nitrogen levels on light intensity under maize, indicate that the highest light intensity was obtained by solid maize and zero $\mathrm{N}$ fed, ${ }^{-1}$ while the lowest value was obtained under intercropping two plants hill ${ }^{-1}$ of cowpea with maize fertilized with $120 \mathrm{~kg} \mathrm{~N} \mathrm{fed}^{-1}$

\subsubsection{The relationship between light intensity and maize grain yield}

Intercropping patterns significantly affected grain yield of maize except when maize was intercropped with two plants hill ${ }^{-1}$ of cowpea and solid maize in both seasons. At the same time light intensity at the top of maize plants before the $1^{\text {st }}$ cut of cowpea (one plant hill ${ }^{-1}$ ) was higher by $0.3 \%$ compared with solid maize. While, light intensity of solid maize increased by $0.1 \%$ compared to intercropping two plants hill ${ }^{-1}$ of cowpea with maize in the first season (Table 10). These results are in agreement with those obtained by Metwally et al.(2009) and disagree with Searle et al. (1981). 
Table (10): Effect of intercropping pattern and nitrogen levels on light intensity (lux) at the top of maize plants before the first cut of forage cowpea in 2008 and 2009.

\begin{tabular}{|c|c|c|c|c|c|c|c|c|c|c|}
\hline \multirow{3}{*}{$\begin{array}{l}\text { Intercropping } \\
\text { Pattern }\end{array}$} & \multicolumn{5}{|c|}{2008} & \multicolumn{5}{|c|}{2009} \\
\hline & \multicolumn{4}{|c|}{ Nitrogen levels $\left(\mathrm{kg} \mathrm{fed}^{-1}\right)$} & \multirow{2}{*}{ Mean } & \multicolumn{4}{|c|}{ Nitrogen levels $\left(\mathrm{kg} \mathrm{fed}^{-1}\right)$} & \multirow{2}{*}{ Mean } \\
\hline & Zero & 60 & 90 & 120 & & Zero & 60 & 90 & 120 & \\
\hline Solid Maize & 1593.0 & 1583.0 & 1578.0 & 1574.0 & 1582.0 & 1603.0 & 1596.0 & 1592.0 & 1581.0 & 1593.0 \\
\hline Maize + Cowpea (one plant hill ${ }^{-1}$ ) & 1595.0 & 1590.0 & 1583.0 & 1580.0 & 1587.0 & 1598.0 & 1593.0 & 1590.0 & 1583.0 & 1591.0 \\
\hline Maize + Cowpea (two plants hill ${ }^{-1}$ ) & 1591.0 & 1580.0 & 1576.0 & 1573.0 & 1580.0 & 1596.0 & 1592.0 & 1581.0 & 1575.0 & 1586.0 \\
\hline Mean & 1593.0 & 1584.3 & 1579.0 & 1575.7 & 1583.0 & 1599.0 & 1593.7 & 1587.7 & 1579.7 & 1590.0 \\
\hline \multicolumn{11}{|l|}{ L.S.D. at 0.05 level: } \\
\hline Intercropping pattern $(\mathrm{A})$ & \multicolumn{5}{|c|}{4.1} & \multicolumn{5}{|c|}{8.7} \\
\hline Nitrogen levels (B) & \multicolumn{5}{|c|}{3.6} & \multicolumn{5}{|c|}{3.5} \\
\hline $\mathrm{A} \times \mathrm{B}$ & \multicolumn{5}{|c|}{6.2} & \multicolumn{5}{|c|}{6.1} \\
\hline
\end{tabular}

Table (11): Effect of intercropping patterns and nitrogen levels on light intensity (lux) at the top of maize plants before the second cut of forage cowpea 2008 and 2009.

\begin{tabular}{|c|c|c|c|c|c|c|c|c|c|c|}
\hline \multirow{3}{*}{$\begin{array}{l}\text { Intercropping } \\
\text { Pattern }\end{array}$} & \multicolumn{5}{|c|}{2008} & \multicolumn{5}{|c|}{2009} \\
\hline & \multicolumn{4}{|c|}{ Nitrogen levels $\left(\mathrm{kg} \mathrm{fed}^{-1}\right)$} & \multirow{2}{*}{ Mean } & \multicolumn{4}{|c|}{ Nitrogen levels $\left(\mathrm{kg} \mathrm{fed}^{-1}\right)$} & \multirow{2}{*}{ Mean } \\
\hline & Zero & 60 & 90 & 120 & & Zero & 60 & 90 & 120 & \\
\hline Solid Maize & 1592.0 & 1583.0 & 1578.0 & 1567.0 & 1580.0 & 1600.0 & 1595.0 & 1588.0 & 1569.0 & 1588.0 \\
\hline Maize + Cowpea (one plant hill ${ }^{-1}$ ) & 1581.0 & 1576.0 & 1573.0 & 1566.0 & 1574.0 & 1597.0 & 1590.0 & 1584.0 & 1569.0 & 1585.0 \\
\hline Maize + Cowpea (two plants hill ${ }^{-1}$ ) & 1588.0 & 1580.0 & 1574.0 & 1570.0 & 1578.0 & 1591.0 & 1586.0 & 1580.0 & 1575.0 & 1583.0 \\
\hline Mean & 1587.0 & 1579.0 & 1575.0 & 1567.7 & 1577.3 & 1596.0 & 1590.3 & 1584.0 & 1571.0 & 1585.3 \\
\hline \multicolumn{11}{|l|}{ L.S.D. at 0.05 level: } \\
\hline Intercropping pattern (A) & \multirow{2}{*}{\multicolumn{5}{|c|}{4.5}} & \multicolumn{5}{|c|}{2.5} \\
\hline Nitrogen levels (B) & \multicolumn{3}{|r|}{4.3} & & & \multicolumn{5}{|c|}{4.7} \\
\hline $\mathrm{A} \times \mathrm{B}$ & \multicolumn{5}{|c|}{7.4} & \multicolumn{5}{|c|}{8.1} \\
\hline
\end{tabular}

Table (12): Effect of intercropping pattern and nitrogen levels on light intensity (lux) at the middle of maize plants before the $1^{\text {st }}$ cut of forage cowpea 2008 and 2009.

\begin{tabular}{|c|c|c|c|c|c|c|c|c|c|c|}
\hline \multirow{3}{*}{$\begin{array}{l}\text { Intercropping } \\
\text { Pattern }\end{array}$} & \multicolumn{5}{|c|}{2008} & \multicolumn{5}{|c|}{2009} \\
\hline & \multicolumn{4}{|c|}{ Nitrogen levels $\left(\mathrm{kg} \mathrm{fed}^{-1}\right)$} & \multirow{2}{*}{ Mean } & \multicolumn{4}{|c|}{ Nitrogen levels $\left(\mathrm{kg} \mathrm{fed}^{-1}\right)$} & \multirow{2}{*}{ Mean } \\
\hline & Zero & 60 & 90 & 120 & & Zero & 60 & 90 & 120 & \\
\hline Solid Maize & 985.0 & 971.0 & 954.0 & 946.0 & 964.0 & 987.0 & 968.0 & 958.0 & 931.0 & 961.0 \\
\hline Maize + Cowpea (one plant hill' ${ }^{-1}$ ) & 953.0 & 936.0 & 924.0 & 915.0 & 932.0 & 958.0 & 936.0 & 912.0 & 902.0 & 927.0 \\
\hline Mean & 958.3 & 942.7 & 929.3 & 919.0 & 937.3 & 955.3 & 938.0 & 922.0 & 907.3 & 930.7 \\
\hline \multicolumn{11}{|l|}{ L.S.D. at 0.05 level: } \\
\hline Intercropping pattern (A) & \multicolumn{5}{|c|}{5.1} & \multicolumn{5}{|c|}{3.6} \\
\hline Nitrogen levels (B) & \multicolumn{5}{|c|}{3.5} & \multicolumn{5}{|c|}{4.4} \\
\hline
\end{tabular}


Table (13): Effect of intercropping pattern and nitrogen levels on light intensity (lux) at the middle of maize plants before the $2^{\text {nd }}$ cut of forage cowpea during 2008 and 2009.

\begin{tabular}{|c|c|c|c|c|c|c|c|c|c|c|}
\hline \multirow{3}{*}{$\begin{array}{l}\text { Intercropping } \\
\text { Pattern }\end{array}$} & \multicolumn{5}{|c|}{2008} & \multicolumn{5}{|c|}{2009} \\
\hline & \multicolumn{4}{|c|}{ Nitrogen levels $\left(\mathrm{kg} \mathrm{fed}^{-1}\right)$} & \multirow{2}{*}{ Mean } & \multicolumn{4}{|c|}{ Nitrogen levels $\left(\mathrm{kg} \mathrm{fed}^{-1}\right)$} & \multirow{2}{*}{ Mean } \\
\hline & Zero & 60 & 90 & 120 & & Zero & 60 & 90 & 120 & \\
\hline Solid Maize & 968.0 & 955.0 & 941.0 & 936.0 & 950.0 & 973.0 & 950.0 & 941.0 & 936.0 & 950.0 \\
\hline Maize + Cowpea (one plant hill' ${ }^{-1}$ ) & 942.0 & 931.0 & 917.0 & 914.0 & 926.0 & 933.0 & 921.0 & 900.0 & 890.0 & 911.0 \\
\hline Maize + Cowpea (two plants hill ${ }^{-1}$ ) & 927.0 & 910.0 & 897.0 & 894.0 & 907.0 & 910.0 & 896.0 & 884.0 & 878.0 & 892.0 \\
\hline Mean & 945.7 & 932.0 & 918.3 & 914.7 & 927.7 & 938.7 & 922.3 & 908.3 & 901.3 & 917.7 \\
\hline \multicolumn{11}{|l|}{ L.S.D. at 0.05 level: } \\
\hline Intercropping pattern $(\mathrm{A})$ & \multicolumn{5}{|c|}{3.4} & \multicolumn{5}{|c|}{6.9} \\
\hline Nitrogen levels (B) & \multicolumn{5}{|c|}{3.8} & \multicolumn{5}{|c|}{4.1} \\
\hline $\mathrm{A} \times \mathrm{B}$ & \multicolumn{5}{|c|}{6.5} & \multicolumn{5}{|c|}{7.2} \\
\hline
\end{tabular}

Table (14): Effect of intercropping pattern and nitrogen levels on light intensity (lux) at the bottom of maize plants before the $1{ }^{\text {st }}$ cut of forage cowpea during 2008 and 2009.

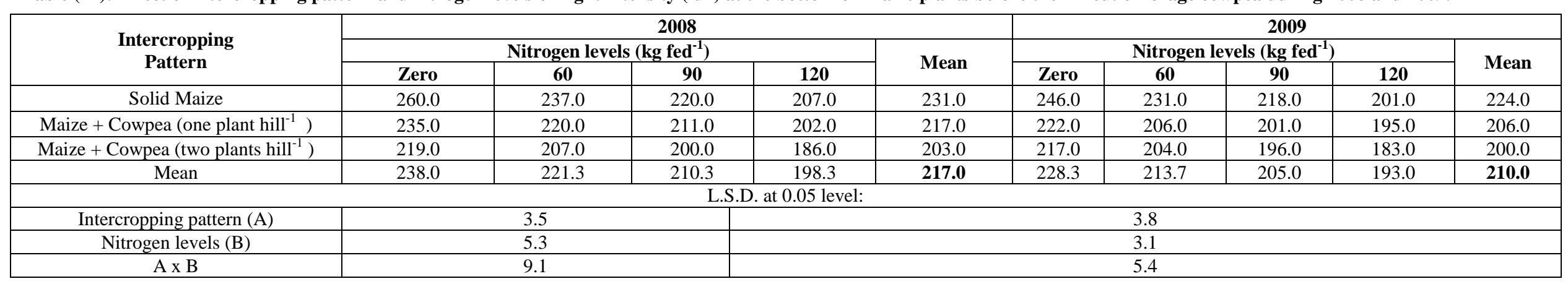

Table (15): Effect of intercropping pattern and nitrogen levels on light intensity (lux) at the bottom of maize plants before the $2^{\text {nd }}$ cut of forage cowpea during 2008 and 2009.

\begin{tabular}{|c|c|c|c|c|c|c|c|c|c|c|}
\hline \multirow{3}{*}{$\begin{array}{l}\text { Intercropping } \\
\text { Pattern }\end{array}$} & \multicolumn{5}{|c|}{2008} & \multicolumn{5}{|c|}{2009} \\
\hline & \multicolumn{4}{|c|}{ Nitrogen levels $\left(\mathrm{kg} \mathrm{fed}^{-1}\right)$} & \multirow{2}{*}{ Mean } & \multicolumn{4}{|c|}{ Nitrogen levels $\left(\mathrm{kg} \mathrm{fed}^{-1}\right)$} & \multirow{2}{*}{ Mean } \\
\hline & Zero & 60 & 90 & 120 & & Zero & 60 & 90 & 120 & \\
\hline Solid Maize & 239.0 & 227.0 & 219.0 & 195.0 & 220.0 & 221.0 & 213.0 & 210.0 & 196.0 & 210.0 \\
\hline Maize + Cowpea (one plant hill ${ }^{-1}$ ) & 220.0 & 208.0 & 198.0 & 194.0 & 205.0 & 209.0 & 196.0 & 189.0 & 182.0 & 194.0 \\
\hline Maize + Cowpea (two plants hill ${ }^{-1}$ ) & 201.0 & 194.0 & 190.0 & 187.0 & 193.0 & 208.0 & 196.0 & 180.0 & 172.0 & 189.0 \\
\hline Mean & 220.0 & 209.7 & 202.3 & 192.0 & 206.0 & 212.7 & 201.7 & 193.0 & 183.3 & 197.7 \\
\hline \multicolumn{11}{|l|}{ L.S.D. at 0.05 level: } \\
\hline Intercropping pattern (A) & \multicolumn{5}{|c|}{1.9} & \multicolumn{5}{|c|}{4.8} \\
\hline Nitrogen levels (B) & \multicolumn{5}{|c|}{5.5} & \multicolumn{5}{|c|}{3.9} \\
\hline $\mathrm{A} \times \mathrm{B}$ & \multicolumn{5}{|c|}{9.6} & \multicolumn{5}{|c|}{6.9} \\
\hline
\end{tabular}


Table (16): Grain yield $\left(\mathrm{t} \mathrm{fed}^{-1}\right)$ of maize as affected by intercropping pattern and nitrogen levels during 2008 and 2009.

\begin{tabular}{|c|c|c|c|c|c|c|c|c|c|c|}
\hline \multirow{3}{*}{$\begin{array}{c}\text { Intercropping } \\
\text { Pattern }\end{array}$} & \multicolumn{5}{|c|}{2008} & \multicolumn{5}{|c|}{2009} \\
\hline & \multicolumn{4}{|c|}{ Nitrogen levels $\left(\mathrm{kg} \mathrm{fed}^{-1}\right)$} & \multirow{2}{*}{ Mean } & \multicolumn{4}{|c|}{ Nitrogen levels $\left(\mathrm{kg} \mathrm{fed}^{-1}\right)$} & \multirow{2}{*}{ Mean } \\
\hline & Zero & 60 & 90 & 120 & & Zero & 60 & 90 & 120 & \\
\hline Solid Maize & 3.0 & 3.9 & 4.1 & 4.2 & 3.8 & 2.5 & 3.2 & 3.4 & 3.5 & 3.2 \\
\hline Maize + Cowpea (one plant hill ${ }^{-1}$ ) & 3.3 & 3.9 & 4.2 & 4.3 & 3.9 & 2.8 & 3.3 & 3.5 & 3.6 & 3.3 \\
\hline Maize + Cowpea (two plants hill ${ }^{-1}$ ) & 3.2 & 3.9 & 4.0 & 4.2 & 3.8 & 2.6 & 3.2 & 3.3 & 3.5 & 3.2 \\
\hline Mean & 3.2 & 3.9 & 4.1 & 4.2 & 3.8 & 2.6 & 3.2 & 3.4 & 3.5 & 3.2 \\
\hline \multicolumn{11}{|l|}{ L.S.D. at 0.05 level: } \\
\hline Intercropping pattern (A) & \multicolumn{5}{|c|}{0.1} & \multicolumn{5}{|c|}{0.1} \\
\hline Nitrogen levels (B) & \multicolumn{5}{|c|}{0.1} & \multicolumn{5}{|c|}{0.1} \\
\hline $\mathbf{A} \times \mathbf{B}$ & \multicolumn{5}{|c|}{0.2} & \multicolumn{5}{|c|}{0.2} \\
\hline
\end{tabular}

Table (17): Effect of intercropping patterns and nitrogen levels on stover dry yield (t fed ${ }^{-1}$ ) of maize during 2008 and 2009.

\begin{tabular}{|c|c|c|c|c|c|c|c|c|c|c|}
\hline \multirow{3}{*}{$\begin{array}{l}\text { Intercropping } \\
\text { Pattern }\end{array}$} & \multicolumn{5}{|c|}{2008} & \multicolumn{5}{|c|}{2009} \\
\hline & \multicolumn{4}{|c|}{ Nitrogen levels $\left(\mathrm{kg} \mathrm{fed}^{-1}\right)$} & \multirow{2}{*}{ Mean } & \multicolumn{4}{|c|}{ Nitrogen levels $\left(\mathrm{kg} \mathrm{fed}^{-1}\right)$} & \multirow{2}{*}{ Mean } \\
\hline & Zero & 60 & 90 & 120 & & Zero & 60 & 90 & 120 & \\
\hline Solid Maize & 2.1 & 2.7 & 3.4 & 3.8 & 3.0 & 1.8 & 2.2 & 2.9 & 3.1 & 2.5 \\
\hline Maize + Cowpea (one plant hill $^{-1}$ ) & 2.4 & 3.0 & 3.7 & 4.2 & 3.3 & 1.9 & 2.5 & 3.1 & 3.5 & 2.7 \\
\hline Maize + Cowpea (two plants hill ${ }^{-1}$ ) & 2.8 & 3.5 & 4.6 & 4.5 & 3.8 & 2.3 & 2.9 & 3.7 & 3.8 & 3.2 \\
\hline Mean & 2.4 & 3.1 & 3.9 & 4.2 & 3.4 & 2.0 & 2.5 & 3.2 & 3.5 & 2.8 \\
\hline \multicolumn{11}{|l|}{ L.S.D. at 0.05 level: } \\
\hline Intercropping pattern(A) & \multicolumn{5}{|c|}{0.2} & \multicolumn{5}{|c|}{0.2} \\
\hline Nitrogen levels (B) & \multicolumn{5}{|c|}{0.2} & \multicolumn{5}{|c|}{0.2} \\
\hline$A \times B$ & \multicolumn{5}{|c|}{0.3} & \multicolumn{5}{|c|}{0.3} \\
\hline
\end{tabular}


Nitrogen application significantly affected grain yield of maize when applied at levels below 90 and $120 \mathrm{~kg} \mathrm{~N} f e d^{-1}$ in both seasons.

The data indicate that grain yield of maize increased with increasing nitrogen fertilizer levels from zero to $120 \mathrm{~kg} \mathrm{~N}^{-1} \mathrm{~d}^{-1}$ recording 3.2 to $4.2 \mathrm{t} \mathrm{fed}^{-1}$ in the first season and 2.6 to $3.5 \mathrm{t}$ $\mathrm{fed}^{-1}$ in the second season (Table 16). Nitrogen levels significantly decreased light intensity at the top, middle and bottom levels of maize canopy before the $1^{\text {st }}$ and $2^{\text {nd }}$ cuts of cowpea in both seasons, except between the two levels of 90 and $120 \mathrm{~kg} \mathrm{~N}$ fed $^{-1}$ for the top and middle readings before the $1^{\text {st }}$ and $2^{\text {nd }}$ cuts of cowpea in the first season. The same trend was obtained by (Searle et al., 1981; Baker and Blamey, 1985; Rana et al., 2001 and Safina, 2007).

The interaction between intercropping patterns and nitrogen levels was most favorable when one plant hill ${ }^{-1}$ of cowpea was intercropped with maize and $120 \mathrm{~kg} \mathrm{~N}^{-1} \mathrm{fe}^{-1}$ in both seasons, recording $4.3 \mathrm{t} \mathrm{fed}^{-1}$ in the first season and $3.6 \mathrm{t}$ $\mathrm{fed}^{-1}$ in the second season (Table 16). These results are in agreement with those given by Metwally, (1973). On the other hand, the lowest value was obtained by solid maize and zero nitrogen level in both seasons. It was recorded $3.0 \mathrm{t} \mathrm{fed}^{-1}$ in the first season and $2.5 \mathrm{t} \mathrm{fed}^{-1}$ in the second one (Table 16). These results are in line with Safina, (2007).

\subsubsection{The relationship between light intensity and dry stover yield}

Significant differences between intercropping patterns for dry stover yield $\left(\mathrm{t} \mathrm{fed}^{-1}\right)$ in both seasons, except between the intercropping of one plant hill ${ }^{-1}$ of cowpea with maize, as well as solid maize in the second season. These results are in agreement with those given by Metwally et al. (2009) on the effect of cropping systems and maize varieties on maize intercropped with soybean. The workers found that the cropping system had significant effects on maize dry weight.

The highest dry weight was obtained by intercropping two cowpea plants hill ${ }^{-1}$ with maize in both seasons.

Nitrogen fertilization significantly increased dry maize stover yield in the first and second seasons and reduced light intensity.

Dry stover yield of maize supplied with zero to $120 \mathrm{~kg} \mathrm{~N} \mathrm{fed}^{-1}$ ranged from 2.4 to $4.2 \mathrm{t} \mathrm{fed}^{-1}$ in the first season, 2.0 to $3.5 \mathrm{t} \mathrm{fed}^{-1}$ in the second one. The greater dry stover yield average was obtained by intercropping two cowpea plants hill $^{-1}$ with maize fertilized with $120 \mathrm{~kg} \mathrm{~N} \mathrm{fed}^{-1}$ in both seasons (Table 17).

\section{REFERENCES}

Abdel-Wahab Sh.I. (2010). Growing soybean with maize under different intercropping patterns and high population densities. Ph.D. Thesis, Fac. Agric. Cairo Univ. Egypt, 189 p.

Abou Keriasha M.A., Abd El-Hady M.A. and Nawar F.R. (2009). Response of some cowpea varieties to intercropping in maize fields under upper Egypt conditions. Egypt. J. of Appl. Sci., 24(2B):495-514.

Badr M. M. (1998). Biological efficiency of different intercropping patterns of soybean with maize. Ph. D. Thesis, Fac. Agric., Mansoura Univ., Egypt, 102 p.

Baker C.M. and Blamey F.P.C. (1985). Nitrogen fertilizer effects on yield and nitrogen uptake of sorghum and soybean, grown in sole cropping and intercropping systems. Field Crops Research, 12: 233-240.

Bowes G., Ogren W.L. and Hageman R.H. (1970). Light saturation photosynthetic rate, RuDP carboxylase: activity and specific leaf weight in soybeans grown under different light intensities. Crop Sci., 12: 77-79.

El-Zanaty Rafea I.A. (2006). Intercropping maize with cowpea. 1- Effect of intercropping patterns on forage yield, quality and seed yield of cowpea. Egypt. J. of Appl. Sci., 21(6B):523-534.

Freed R (2005). MSTAT-C Crop and Soil Sciences. Michigan State University,MI, USA.

Li L., Zhang F.S., Li X.L., Christie P., Sun J.H., Yang S.C. and Tang C. (2003). Inter specific facilitation of nutrient uptake by intercropped maize and faba bean. Nutr. Cycling Agro. Eco., 68:61-71.

Marsalis M. and Angadi S. (2009). Can intercropping legumes with sorghum improve sustainability? Past - articles \& Itemid, November, 2009.

Metwally A.A. (1973). The study of some companionship between maize and other crops. M.Sc. Thesis, Fac. Agric., Cairo Univ., Egypt, 151 p.

Metwally A. A., Mohamed Gmalat O., Sherief M. N. and Abo-Hegazy D. R. (2009). Yield and land equivalent ratio of intercropped maize and soybean under three intercropping patterns. The $4^{\text {th }}$ Conf., Recent Technologies in Agric., 3-5 Nov., Cairo Univ., 2: 284-290. 
Metwally A. A., Shafik M.M., El Morshedy W.A. and Aly H.R. (2005). Yield and land equivalent ratio of intercropped maize and soybean. Proc. $1^{\text {st }}$ Sci. Conf. Cereal Crops, Alex., : 113-120.

Ofori F. and Stern W.R. (1987). Cereal-legume intercropping systems. Advance in Agronomy. 41: 41-90.

Rana R.S., Bhupinder S., Negi S.C. and Singh B. (2001). Management of maize/legume intercropping under mid-hill sub-humid conditions. Indian-Journal-of-Agricultural-

Research. 35(2): 100-103.

Safina S.A. (2007). Effect of biofertilization and mineral nitrogen applications on solid and intercropping maize with soybean. Ph.D. Thesis, Fac. Agric., Cairo Univ., Egypt, 151 p.

Searle P.G.E., Comudom Y., Shedden D.C. and Nance R.A. (1981). Effect of maize + legume intercropping systems and fertilizer nitrogen on crop yielding and residual nitrogen. Field Crops Research, 4: 133-145.

العلاقة بين شدة الاضاءة وحاصل العلف الجاف للوبيا العلف والذرة الثامية تحت ظروف التحميل

قرنى اسماعيل عبد الجواد- عبد الحفيظ عبد الرحمن عبد الحفيظ - . وفاء عحم شعراوى *-. ولاء تحمد النبوى*

$$
\begin{aligned}
& \text { قسم المحاصيل ـ كلية الزر اعة ـ جامعة القاهرة. } \\
& \text { * قسم بحوث محاصيل العلف ـ معهد بحوث المحاصيل الحقلية ـ مركز البحوث الزرة اعية ــ الجيزة ـ مصر }
\end{aligned}
$$

\section{ملخص

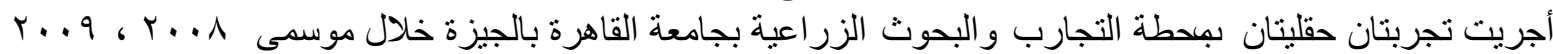

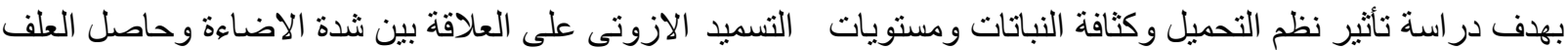

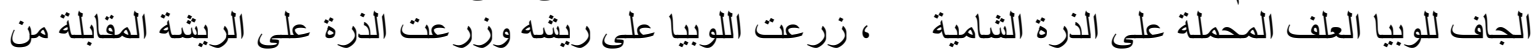

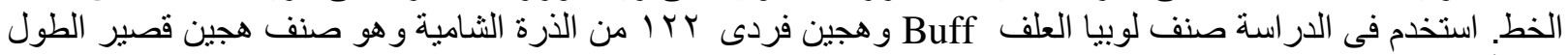

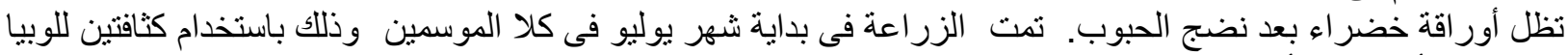

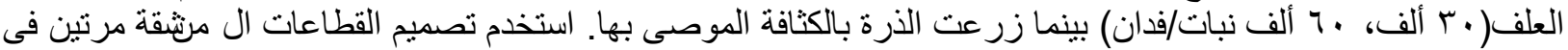

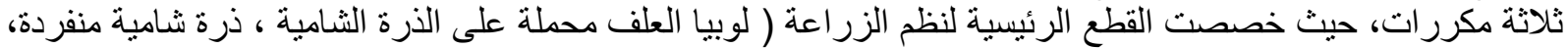

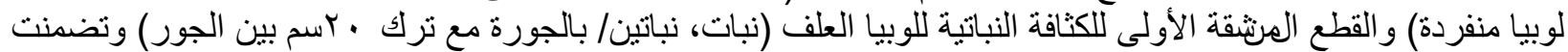

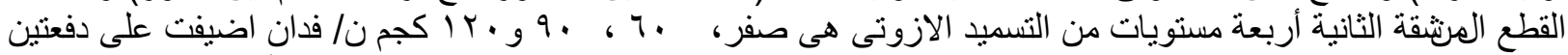

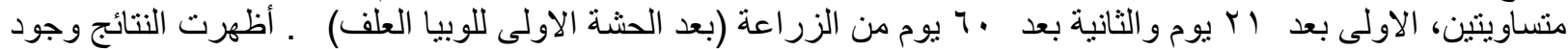

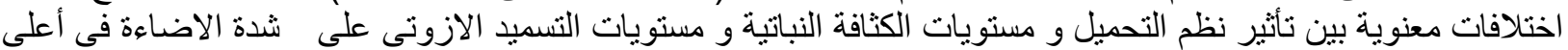

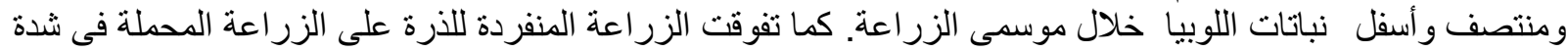

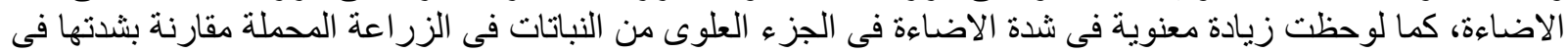

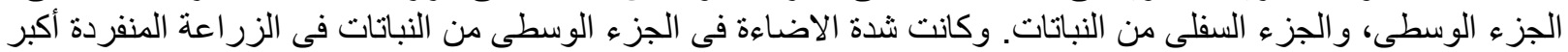

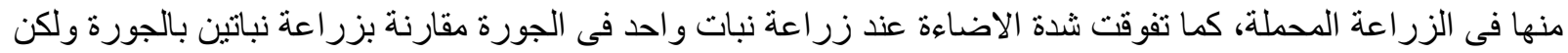

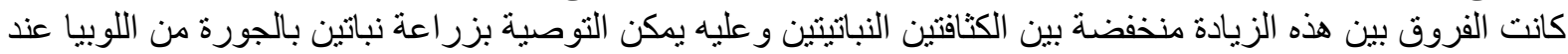

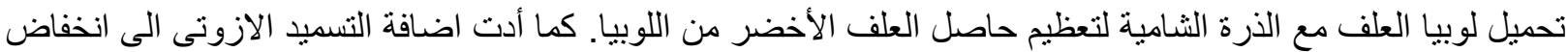

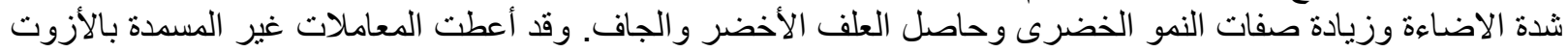

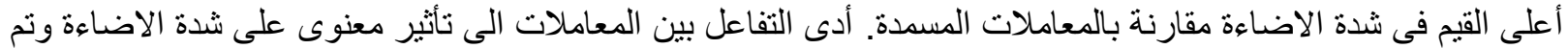

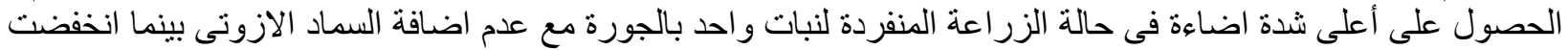

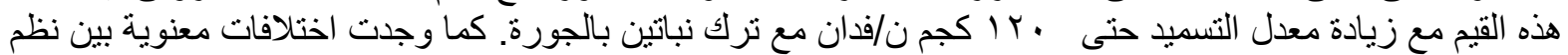

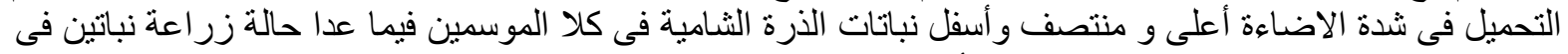

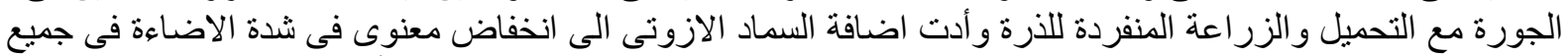

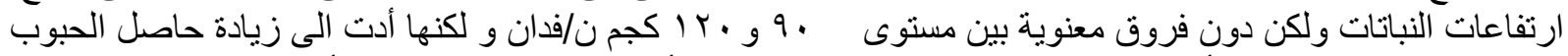

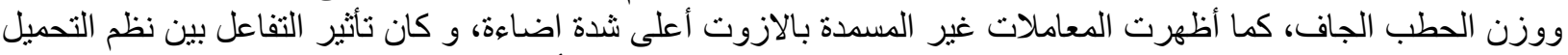

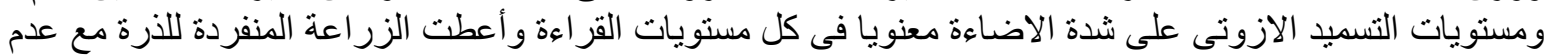

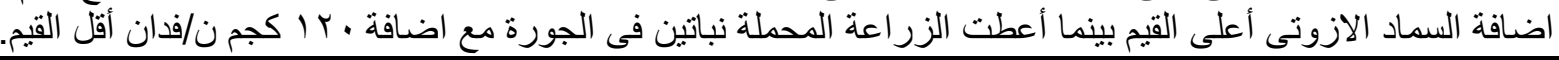

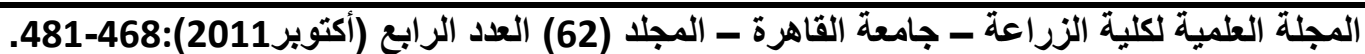

